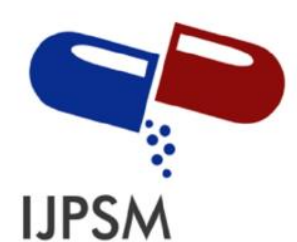

Ting Wan Tan et al, Int. Journal of Pharmaceutical Sciences and Medicine (IJPSM),

Vol.6 Issue. 2, February- 2021, pg. 44-47

ISSN: 2519-9889

Impact Factor: 3.426

\title{
Minimize Risk of Intra-Hospital Acquired Transmission to Confront Pandemic COVID-19 in Taiwan
}

\author{
Ting Wan Tan'; Han Ling Tan³ ${ }^{3}$ Man Na Chang ; Wen Shu Lin ${ }^{1}$; Chih Ming Chang ${ }^{1,2 *}$ \\ 1 Department of Nursing, Hsinchu MacKay Memorial Hospital, Hsinchu 30071, Taiwan; \\ m617@ mmh.org.tw (T.W-T); m008@ mmh.org.tw (M.N-C); wensha@ mmh.org.tw (W.S-L) \\ 2 Graduate Institute of Health Industry Management, Yuanpei University; mackaym617@ gmail.com (C.M-C) \\ 3 Department of Orthopedic Surgery, University Malaya Medical Centre, Kuala Lumpur, Malaysia; \\ hanling6188@gmail.com (H.L-T)
}

DOI: 10.47760/ijpsm.2021.v06i02.005

\begin{abstract}
Since December 2019, the COVID-19 confirmed case number has increased rapidly in China. Subsequently has spread globally. Since late January 2020, Taiwan CDC and government have took measures early and quick response to Covid-19, take action to develop strategies to manage epidemic crisis and community spread in early stage. Taiwan has aware importance of design of isolation route from emergency department, outpatient, intensive care unit, ordinary ward and isolation rooms in health care facilities. For yearly hospital evaluation has been assessed elements that related functional capacity of hospital safety, which aims to examine the level of preparedness of hospital staffs for major emergencies or disaster.
\end{abstract}

Keywords: Intra-hospital; Acquired transmission; COVID-19; Taiwan.

\section{Introduction}

Taiwan has gone through a disastrous experience during the outbreak of SARS in 2002. Taiwan Centers for Disease Control and Prevention has since developed recommendations and guidance of infection prevention and control practices for the country to prevent hospital associated infections in healthcare setting. From the experience, council has started focusing on the importance of the designated 'isolation route' specifically for intra-hospital transfer of infected patients in between wards, from emergency department, outpatient department, ordinary ward, to-and-fro ICU and isolation rooms. Yearly assessment and evaluation will be conducted for the awareness and readiness of the system to anticipate for any outbreaks, comparable to the recent COVID-19. Protocols practiced are as follow:

2. Reorganized hospital entrance and patient flow management

Hospital has designated a separate entrances and exits for suspected inpatients, outpatients and ED patients. Screening staff will request for, access and identify every patient with National Health Insurance smart card to screen for TOCC status(travel, occupation, contact, and cluster), and presence of any suspected symptoms before enter hospital. Authorities have the access of the information, track down infected persons and mapping positive patients according to their district [1]. All staffs, patients and visitors are necessary to undergo temperature check, to wear surgical graded face mask and to perform hand wash before entering the hospital. Patients with positive TOCC history or respiratory illness 


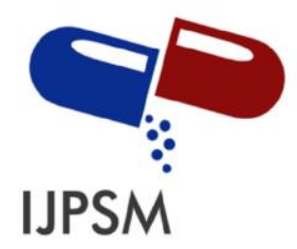

Ting Wan Tan et al, Int. Journal of Pharmaceutical Sciences and Medicine (IJPSM), Vol.6 Issue. 2, February- 2021, pg. 44-47

ISSN: 2519-9889

Impact Factor: 3.426

symptoms will be guide to outdoor Covid-19 special clinic or outdoor emergency triage area for further evaluation, detailed screening and treatment, aimed to reduce risk of hospital acquired infection.

\section{Management control of Emergency Department(ED)}

Outdoor emergency triage area has set up specifically for suspected Covid-19 patients, for further assessment, physical evaluation by physician and undergo Covid-19 PCR test, when necessary. Portable CXR will be arranged for patient in outdoor settings. Unwell Covid-19 cases, patient will be transferred to a designated negative pressure observation rooms in ED, next to triage area. Meanwhile, ED transfer networks team will coordinate and manage patient transfer to isolation ward through separate ED isolation lift, a designated lift to transfer patient, with the shortest route, from ED to isolation ward.

\section{Management control of Outpatient Department (OPD)}

OPD has set up an outdoor registration services for screening purposes. Patient presented with respiratory illness/suspected symptoms/with fever will be guide to outdoor Covid-19 special clinic for further evaluation by physician. Suspected patients whom requires for further Covid-19 PCR test will be guide to outdoor emergency triage area, which is located nearby to Covid-19 special clinic.

\section{Management control of Inpatient Department}

Confirmed Covid-19 cases are restricted within the isolation wards. However, under special circumstances, such as newly suspected inpatients to be transferred to isolation ward or critically ill patient requiring ICU care in isolated ICU [2]. Direct consultant-to-consultant referral to infectious team to evaluate and further discussion with decision to transfer patient. Once decision is made, infectious disease control team will make appropriate arrangement, designate unit will be contacted and do the preparation, isolation network team will follow hospital protocol to transport patient in shortest route, to minimize risk of intra-hospital acquire transmission. Before transfer patient begins, infectious disease control physician will monitor patient's condition and suitability for the transfer. Nurses will prepare adequate supplies to ensure patient safety during intra-hospital transfer. Hospital security staff will go through the planned route earlier and involved corridors will be lock down (with security tape throughout the route), ensure the region is free of other patients and staffs. Special designated transfer members will lead and follow planned route ahead of transport medical team via a designated isolation lift to destination ward as per hospital protocols. Cleaning staff will decontaminate transfer route after transport, controlled route will be decontaminated for 30 minutes. Details of healthcare worker who involved in the transfer will be record for future tracing, if needed.

\section{Disinfection of transport tools and medical equipments}

All transport tools such as: beds, medical equipment involved in the patient transfer, are necessary to be disinfected by well-trained cleaning staff with detergent solution 5000ppm NaHCO3 with disposable cleaning clothes immediately after the transfer. Mobile medical equipment has sent to central sterile services department (CSSD) via separated controlled lift to cleanse and disinfected as CSSD protocols. 


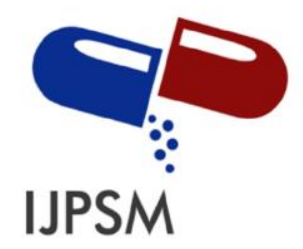

Ting Wan Tan et al, Int. Journal of Pharmaceutical Sciences and Medicine (IJPSM), Vol.6 Issue. 2, February- 2021, pg. 44-47

ISSN: 2519-9889

Impact Factor: 3.426

\section{Ongoing \& update intra-hospital patient transport educations and training}

All involved frontline healthcare workers are required to undergo a compulsory training via online learning resources. Content of such training included COVID-19 triage procedures, COVID-19 case definition, appropriate PPE gowning and doffing. Nursing unit has established handbook for COVID-19 intra-hospital route for patient transfer, recorded education video regarding the various routes of transfer of COVID-19 patients. Special hospital transfer team has take responsibility to, liaise with security team, briefing and, lead transport team to designed unit, prior to the special hospital transfer team will have to run through checklists, to ensure the protocols are being carried out.

\section{Occupational Safety for healthcare workers}

Routine performance protocols have been established to, monitor, and provide appropriate training skills to ensure frontline healthcare workers are aware of while providing care and treatment for patients [3]. Hospital leadership will review the protocols frequently to provide a safe environment and to reduce risk exposure of the frontline healthcare workers.

\section{Conclusion}

Hospital and department leadership is vital to minimize and reduce the exposure risk, to ensure the frontline healthcare workers are aware with the latest information and updates. Regular monitoring and training are essential to maintain staff competencies and improve compliance to the hospital protocols, from patient management to transfer routes. Aim to maintain and achieve an ideal zero healthcare worker infection of Covid-19 during the outbreak. During the process, to prevent the chaos, a special in-charge team will be contactable 24-7 for further assistance of unforeseen circumstances and clarification of the protocols.

Ethics approval and consent to participate: This article belongs to the final editorial and is exempt from ethical review.

Consent for publication: No applicable. Our study contains no individual data.

Availability of supporting data: No applicable.

Competing Interest: The authors declare that they have no competing interests.

Funding: This study was not supported by any of the funding sources.

Author Contributions: All authors have read and approved the final manuscript. T.-W.T., and C.-M.C., has established and writing the manuscript, T.-W.T., C.-M.C., and M.-N.C. designed the study, T.-W.T. wrote the first draft; C.-M.C. had primary responsibility for the final content. H.-L.T. had primary responsibility for the grammar check, proof reading of the manuscript.

Acknowledgments: The authors would thank all frontline healthcare workers for their efforts to combat Covid-19 in Taiwan. 


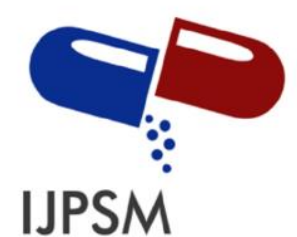

Ting Wan Tan et al, Int. Journal of Pharmaceutical Sciences and Medicine (IJPSM), Vol.6 Issue. 2, February- 2021, pg. 44-47

ISSN: 2519-9889

Impact Factor: 3.426

\section{References}

1. Centers for Disease Control and Prevention, Taiwan, Taiwan: https://www.cdc.gov.tw/En. Assessed on 9 May 2020.

2. Liew, M. F.; Siow, W. T.; Yau, Y. W.; See, K. C. Safe patient transport for COVID-19. Critical Care 2020, $24,1: 94$.

3. Gan, W. H.; Lim, J. W.; Koh, D. Preventing intra-hospital infection and transmission of COVID-19 in healthcare workers. Safety and health at work. 2020, 1-13.

\section{A Brief Author Biography}

Ting Wan Tan has obtained BSC degree from Auckland University of Technology, New Zealand. She is currently worked as a Charge Nurse in Hsinchu Mackay Memorial Hospital; she has published several articles in the finest journal in the area of her studies.

Han Ling Tan has obtained his highest master degree from university of Malaya, Malaysia. He is currently worked as an orthopaedic specialist in Department of Orthopaedic Surgery, University Malaya Medical Centre. He has contributed several articles in the finest journals in the areas of Orthopaedic studies.

Man Na Chang has obtained her highest master degree from Yuanpei University, Taiwan. She is currently worked as a Nurse Supervisor in Hsinchu Mackay Memorial Hospital.

Wen Shu Lin has obtained her BSC degree from Yuanpei University, Taiwan. She is currently worked as a Charge Nurse in Hsinchu Mackay Memorial Hospital. 